\title{
The Impact of Perforator Number on Deep Inferior Epigastric Perforator Flap Breast Reconstruction
}

\author{
Ritwik Grover, Jonas A Nelson, John P Fischer, Stephen J Kovach, Joseph M Serletti, \\ Liza C Wu \\ Division of Plastic Surgery, Hospital of the University of Pennsylvania, Philadelphia, PA, USA
}

Background Perforator flaps minimize abdominal site morbidity during autologous breast reconstruction. The purpose of this study was to assess whether the number of perforators harvested influences the overall deep inferior epigastric perforator (DIEP) flap survival and flap-related complications.

Methods A retrospective review was performed of all DIEP flaps performed at the Hospital of the University of Pennsylvania from 2006 to 2011. The outcomes assessed included flap loss and major complications. We compared flaps by the number of total perforators (1-4) and then carried out a subgroup analysis comparing flaps with one perforator to flaps with multiple perforators. Lastly, we conducted a post-hoc analysis based on body mass index (BMI) categorization.

Results Three hundred thirty-three patients underwent 395 DIEP flaps. No significant differences were noted in the flap loss rate or the overall complications across perforator groups. However, the subgroup analysis revealed significantly higher rates of fat necrosis in the case of one-perforator flaps than in the case of multiple-perforator flaps $(10.2 \% \mathrm{vs} .3 .1 \%$, $\mathrm{P}=0.009$ ). The post-hoc analysis revealed a significant increase in the flap loss rate with increasing BMI $(<30=2.0 \%, 30-34.9=3.1 \%, 35-39.9=3.1 \%,>40=42.9 \%, \mathrm{P}<0.001)$ in the DIEP flaps, but no increase in fat necrosis.

Conclusions This study demonstrates that the number of perforators does not impact the rate of flap survival. However, the rate of fat necrosis may be significantly higher in DIEP flaps based on a single perforator. Multiple perforators should be utilized if possible to decrease the risk of fat necrosis.

Keywords Breast reconstruction / Outcome assessment (health care) / Microsurgery
Correspondence: Liza C Wu Division of Plastic Surgery, Hospital of the University of Pennsylvania, 10 Penn Tower, 3400 Spruce St., Philadelphia, PA 19104, USA

Tel: +1-215-662-2042

Fax: +1-215-349-5995

E-mail: liza.wu@uphs.upenn.edu
The authors would like to express their sincere gratitude to Nancy Folsom BSN for assistance with and preparation for the IRB review.

This article was presented at the American Society for Reconstructive Microsurgery Annual Meeting 2013, January 14, 2013 in Naples, FL, USA

No potential conflict of interest relevant to this article was reported.

Received: 21 Oct 2013 • Revised: 20 Nov 2013 Accepted: 3 Dec 2013

pISSN: 2234-6163 • elSSN: 2234-6171 • http://dx.doi.org/10.5999/aps.2014.41.1.63 • Arch Plast Surg 2014;41:63-70

\section{INTRODUCTION}

An estimated 211,000 new cases of breast cancer are diagnosed each year in the United States, and the rate of post-mastectomy breast reconstruction doubled from 13\% to 26\% between 1998 and 2007 due to multiple factors [1]. The number of patients seeking reconstruction will continue to rise as patients become more aware of their options, and as reconstructive efforts continue to focus on increasing aesthetic outcomes with less donor site morbidity.

Autologous breast reconstruction has undergone an evolution from the free transverse rectus abdominis myocutaneous 
(TRAM) flap to the less invasive deep inferior epigastric perforator (DIEP) flap. The DIEP flap has become a method for autologous breast reconstruction preferred by many surgeons. The benefits of DIEP flaps include tissue that mimics breast suppleness, color, texture, warmth, and ptotic disposition. Furthermore, DIEP flap reconstruction results in a near-negligible abdominal herniation rate of $0.8 \%$, as compared to $3.9 \%$ after free TRAM flaps [2]. Patients undergoing DIEP flap reconstruction are said to experience less donor-site morbidity, reduced postoperative pain, more rapid recovery, and potentially a better postoperative abdominal wall strength profile [3-5].

However, there are downsides to DIEP flaps. The learning curve is steep because dissection around flap perforators can be complex, and complications can occur [6]. The surgeon must balance the number of perforators harvested to adequately perfuse the flap with the amount of intramuscular dissection, taking care not to increase abdominal wall morbidity [7]. This can render DIEP flaps vulnerable to higher rates of fat necrosis when compared to free TRAM flaps (5\%-25\% vs. 6\%-9\%) [8-13].

In reality, plastic surgeons often use their judgement when deciding on the number of perforators required to adequately perfuse the flap while minimizing abdominal wall trauma. Yet, there is a paucity of data that can be used for analyzing the association between the number of perforators and the overall flap perfusion as manifested by flap loss and overall complications. The purpose of this study was to critically examine DIEP flap perfusion to better clarify the association between the number of perforators and DIEP flap breast reconstruction outcomes.

\section{METHODS}

A retrospective review of the prospectively maintained free-flap database at the Hospital of the University of Pennsylvania was performed. Patients who underwent free-flap breast reconstruction between 2006 and 2011 were included for the analysis. We identified all of the patients who underwent DIEP flap reconstruction. This included unilateral cases and bilateral cases of DIEP reconstruction as well as cases in which DIEP flaps were used in combination with either a muscle-sparing free (msf)TRAM or a superficial inferior epigastric artery (SIEA) flap for a bilateral reconstruction.

A detailed review of hospital and office records included the following: preoperative history and physical, operative reports, anesthesia records, postoperative nursing records, discharge summaries, outpatient clinic notes, and laboratory data.

The specific variables examined included baseline patient characteristics (age, body-mass index [BMI], resting heart rate, resting blood pressure, hypertension, chronic obstructive pulmonary disease, hyperlipidemia, active smoking, coronary artery disease, and peripheral arterial disease), and oncologic history (mastectomy type, preoperative and postoperative chemotherapy, and prior radiation). Reconstructive details (intraoperative blood loss, immediate versus delayed, unilateral versus bilateral, thrombotic events, and flap type), intraoperative complications (venous or arterial thrombosis), postoperative surgical complications (flap loss and delayed breast or donor site wound complications), early infection (during hospitalization), delayed infection (outpatient infection), and seroma were examined.

Delayed wound healing at the abdominal donor site and mastectomy skin was defined as skin necrosis or wound breakdown necessitating topical care or dressing changes for more than three weeks. Fat necrosis was defined as a palpable firmness greater than $1 \mathrm{~cm}$ in diameter. This was a clinical finding and not based upon further imaging studies. Partial flap loss was defined as flap loss or atrophy up to $50 \%$ but not requiring an immediate return to the operating room. Superficial infection was defined as incisional cellulitis at either the donor site or the breast that occurred within 30 days.

The database was queried for demographics, past medical history, intraoperative events, flap characteristics, and postoperative flap outcomes. Intraoperative characteristics included thrombotic events and the number of DIEP perforators. Unfortunately, the DIEP perforator numbers were not recorded in the operative notes for some DIEP flaps. Postoperative outcomes included flap loss, postoperative thrombosis, and fat necrosis. The overall outcomes for all of the DIEP flaps were examined.

The following comparisons were performed: Flaps with perforator numbers were compared to those where the number of perforators was not recorded. Flaps with known perforator numbers were then examined, comparing demographics and outcomes by the number of perforators (four cohorts, based on 1-4 perforators). Additionally, we performed a subgroup analysis comparing flaps with one perforator to those with more than one perforator. Finally, in a post-hoc analysis, we analyzed DIEP flap major outcomes by the degree of obesity (World Health Organization [WHO] classification). This included an analysis of flap loss and fat necrosis. Furthermore, fat necrosis was examined by comparing within WHO obesity classifications in an effort to control for the volume of tissue transferred per patient. The WHO obesity class was utilized as a proxy for the volume of the flap, given that flap weight is not routinely measured intraoperatively.

All data were entered into an Excel spreadsheet, and a statistical analysis was performed utilizing STATA IC 10.1. Categorical variables were analyzed using the chi-squared or Fisher's exact tests where appropriate, while continuous variables were exam- 
ined with the Wilcoxon rank sum test or the Kruskal-Wallis test. The statistical significance was set at $\mathrm{P}<0.05$. The results were reported as number, percentage, or median (range). This study was approved by the Institutional Review Board of the Hospital of the University of Pennsylvania.

\section{RESULTS}

Three hundred thirty-three patients underwent a total of 395 DIEP flaps at our tertiary care center between 2006 and 2011. The patient demographic data for these flaps are shown in Table 1. 136 DIEP flaps (34.4\%) were used in unilateral reconstruction, while the remaining 259 flaps $(65.6 \%)$ were used in bilateral reconstruction. 10 of the 395 DIEP flaps (2.5\%) underwent total flap loss. 14 flaps (3.5\%) were noted to have an episode of intraoperative arterial thrombosis. 2 flaps $(0.5 \%)$ had an instance of intraoperative venous thrombosis. 6 flaps (1.5\%) were found to have postoperative arterial thrombosis, while 6 others (1.5\%) were found to have postoperative venous thrombosis. Lastly, fat necrosis was noted in 26 flaps overall (6.6\%) (Table 1).

Overall, the perforator number was obtained in 310 of the 395 flaps included in this study. Comparing patients with a known number of perforators $(310,78.5 \%)$ with those with an unknown number of perforators $(85,21.5 \%)$ revealed that there were no significant differences between the two cohorts with regard to demographic data, past medical history, bilateral reconstruction frequency, or flap outcomes (Table 1).

The 310 DIEP flaps with known perforator numbers were then divided into cohorts according to the number of perforators (1-4) (Table 2). 147 flaps (47.4\%) had 1 perforator, 104 flaps (33.6\%) had 2 perforators, 50 flaps (16.1\%) had 3 perforators, and 9 flaps $(2.9 \%)$ had 4 perforators. Demographic data did not differ significantly between the cohorts except for average BMI. Flaps with 2 or 3 perforators were associated with patients with slightly lower BMI $(\mathrm{P}=0.04)$.

The outcomes assessed included total flap loss and overall complications including intraoperative arterial and venous thrombosis, postoperative arterial and venous thrombosis, and fat necrosis (Table 2). 8 cases of total flap loss occurred in this cohort of 310 DIEP flaps for an overall flap loss rate of $2.6 \% .5$ cases of flap loss occurred in the 1-perforator group (3.4\%), 2 cases in the 2-perforator group (1.9\%), and 1 case of flap loss in the 3-perforator group (2.0\%). No flaps were lost in the 4-perforator group. When examining the flap loss rate across all perforator groups, no significant differences were noted $(P=0.83)$.

Nine flaps suffered intraoperative arterial thrombosis for an overall rate of $2.9 \%$. 4 of the thrombotic events occurred in the 1-perforator group (2.7\%) and 5 cases in the 2-perforator group (4.8\%). No intraoperative arterial thrombotic events occurred in the 3- or 4-perforator groups $(\mathrm{P}=0.37) .2$ flaps suffered intra-

\section{Table 1. Overall patient characteristics a comparison of flaps based on known perforator numbers}

\begin{tabular}{|c|c|c|c|c|}
\hline \multirow{2}{*}{ Characteristic } & \multirow{2}{*}{$\begin{array}{l}\text { Total flaps } \\
(n=395)\end{array}$} & \multicolumn{3}{|c|}{ DIEP flaps with known perforator numbers } \\
\hline & & No $(n=85)$ & Yes $(n=310)$ & P-value \\
\hline \multicolumn{5}{|l|}{ Patient characteristics (n, \%) } \\
\hline Age (yr) (range) & $49.5(20-73)$ & 50.5 (27-68) & $49.0(20-73)$ & 0.83 \\
\hline BMI $\left(\mathrm{kg} / \mathrm{m}^{2}\right)$ (range) & $27.5(19.7-46.3)$ & $26.76(20.5-43.3)$ & $28.1(19.7-46.3)$ & 0.31 \\
\hline Obesity & $121(30.6)$ & $19(22.4)$ & $102(32.9)$ & 0.10 \\
\hline WHO classification & & & & 0.61 \\
\hline 0 & $254(64.3)$ & $50(58.8)$ & $204(65.8)$ & \\
\hline । & $80(20.3)$ & $16(18.8)$ & $64(20.6)$ & \\
\hline$\|$ & $36(9.1)$ & $4(4.7)$ & $32(10.3)$ & \\
\hline III & $8(2.0)$ & $1(1.2)$ & $7(2.3)$ & \\
\hline Diabetes & $26(6.6)$ & $4(4.7)$ & $22(7.1)$ & 0.58 \\
\hline Hypertension & $103(26.1)$ & $20(23.5)$ & $83(26.8)$ & 0.55 \\
\hline Hyperlipidemia & $66(16.7)$ & $12(14.1)$ & $54(17.4)$ & 0.55 \\
\hline Unilateral procedure & $143(36.2)$ & $33(38.8)$ & $110(35.5)$ & 0.57 \\
\hline Bilateral procedure & $252(63.8)$ & $52(61.2)$ & $200(64.5)$ & \\
\hline \multicolumn{5}{|l|}{ Outcomes (n, \%) } \\
\hline Flap loss & $10(2.5)$ & $2(2.4)$ & $8(2.6)$ & 1.00 \\
\hline Intraoperative arterial thrombosis & $14(3.5)$ & $5(5.9)$ & $9(2.9)$ & 0.19 \\
\hline Intraoperative venous thrombosis & $2(0.5)$ & $0(0.0)$ & $2(0.6)$ & 1.00 \\
\hline Postoperative arterial thrombosis & $6(1.5)$ & $2(2.4)$ & $4(1.3)$ & 0.61 \\
\hline Postoperative venous thrombosis & $6(1.5)$ & $1(1.2)$ & $5(1.6)$ & 1.00 \\
\hline Fat necrosis & $28(7.1)$ & $6(7.1)$ & $22(7.1)$ & 0.80 \\
\hline
\end{tabular}


Table 2. Analysis of DIEP flaps by number of perforators

\begin{tabular}{|c|c|c|c|c|c|}
\hline \multirow{2}{*}{ Characteristic } & \multicolumn{4}{|c|}{ Cohorts based on the number of perforators to DIEP $(n=310)$} & \multirow{2}{*}{ P-value } \\
\hline & $1(n=147)$ & $2(n=104)$ & $3(n=50)$ & $4(n=9)$ & \\
\hline \multicolumn{6}{|l|}{ Patient characteristics (n, \%) } \\
\hline Age (yr) (range) & $49.0(20-73)$ & $49(34-73)$ & $52(32-68)$ & $52(40-73)$ & 0.44 \\
\hline BMl $\left(\mathrm{kg} / \mathrm{m}^{2}\right)$ (range) & $28.9(21-46.3)$ & $26.6(19.7-45.3)$ & $27.4(20.6-39.1)$ & $29.0(25.7-36.7)$ & 0.04 \\
\hline Obesity & $54(36.7)$ & $27(26.0)$ & $19(38.0)$ & $2(22.2)$ & 0.24 \\
\hline WHO classification & & & & & 0.75 \\
\hline 0 & $93(63.3)$ & $74(71.2)$ & $31(62.0)$ & $6(66.7)$ & \\
\hline । & $34(23.1)$ & $15(14.4)$ & $13(26.0)$ & $2(22.2)$ & \\
\hline$\|$ & $16(10.9)$ & $9(8.7)$ & $6(12.0)$ & $1(11.1)$ & \\
\hline III & $4(2.7)$ & $3(2.9)$ & $0(0.0)$ & $0(0.0)$ & \\
\hline Diabetes & $8(5.4)$ & $5(4.8)$ & $8(16.0)$ & $1(11.1)$ & 0.06 \\
\hline Hypertension & $41(27.9)$ & $23(22.1)$ & $17(34.0)$ & $2(22.2)$ & 0.45 \\
\hline Hyperlipidemia & $27(18.4)$ & $12(11.5)$ & $13(26.0)$ & $2(22.2)$ & 0.15 \\
\hline Unilateral procedure & $50(34.0)$ & $35(33.7)$ & $22(44.0)$ & $3(33.3)$ & 0.60 \\
\hline Bilateral procedure & $97(66.0)$ & $69(66.3)$ & $28(56.0)$ & $6(66.7)$ & \\
\hline \multicolumn{6}{|l|}{ Outcomes (n, \%) } \\
\hline Flap loss & $5(3.4)$ & $2(1.9)$ & $1(2.0)$ & $0(0.0)$ & 0.83 \\
\hline Intraoperative arterial thrombosis & $4(2.7)$ & $5(4.8)$ & $0(0.0)$ & $0(0.0)$ & 0.37 \\
\hline Intraoperative venous thrombosis & $2(1.4)$ & $0(0.0)$ & $0(0.0)$ & $0(0.0)$ & 0.53 \\
\hline Postoperative arterial thrombosis & $3(2.0)$ & $1(1.0)$ & $0(0.0)$ & $0(0.0)$ & 0.70 \\
\hline Postoperative venous thrombosis & $3(2.0)$ & $2(1.9)$ & $0(0.0)$ & $0(0.0)$ & 0.75 \\
\hline Fat necrosis & $15(10.2)$ & $3(2.9)$ & $2(4.0)$ & $0(0.0)$ & 0.10 \\
\hline
\end{tabular}

\section{Table 3. DIEP subgroup analysis}

\begin{tabular}{|c|c|c|c|}
\hline \multirow[b]{2}{*}{ Characteristic } & \multicolumn{2}{|c|}{ DIEP flaps } & \multirow[b]{2}{*}{ P-value } \\
\hline & $\begin{array}{l}1 \text { Perforator } \\
(n=147)\end{array}$ & $\begin{array}{c}>1 \text { Perforator } \\
(n=163)\end{array}$ & \\
\hline \multicolumn{4}{|l|}{ Patient characteristics (n, \%) } \\
\hline Age (yr) & $49.0(20-73)$ & $50.0(32-73)$ & 0.21 \\
\hline BMI $\left(\mathrm{kg} / \mathrm{m}^{2}\right)$ & $28.9(21-46.3)$ & $27.2(19.7-45.3)$ & 0.04 \\
\hline Obesity & $54(36.7)$ & $48(29.4)$ & 0.17 \\
\hline WHO classification & & & 0.07 \\
\hline 0 & $93(63.3)$ & $111(68.1)$ & \\
\hline I & $34(23.1)$ & $30(18.4)$ & \\
\hline$\|$ & $16(10.9)$ & $16(9.8)$ & \\
\hline III & $4(2.7)$ & $3(1.8)$ & \\
\hline Diabetes & $8(5.4)$ & $14(8.6)$ & 0.28 \\
\hline Hypertension & $41(27.9)$ & $42(25.8)$ & 0.67 \\
\hline Hyperlipidemia & $27(18.4)$ & 27 (16.6) & 0.68 \\
\hline Unilateral procedure & $50(34.0)$ & $60(36.8)$ & 0.61 \\
\hline Bilateral procedure & $97(66.0)$ & $103(63.2)$ & \\
\hline \multicolumn{4}{|l|}{ Outcomes (n, \%) } \\
\hline Flap loss & $5(3.4)$ & $3(1.8)$ & 0.39 \\
\hline Intraoperative arterial thrombosis & $4(2.7)$ & $5(3.1)$ & 0.86 \\
\hline Intraoperative venous thrombosis & $2(1.4)$ & $0(0.0)$ & 0.14 \\
\hline Postoperative arterial thrombosis & $3(2.0)$ & $1(0.6)$ & 0.35 \\
\hline Postoperative venous thrombosis & $3(2.0)$ & $2(1.2)$ & 0.67 \\
\hline Fat necrosis & 15 (10.2) & $5(3.1)$ & 0.009 \\
\hline
\end{tabular}

operative venous thrombosis, both occurring in the 1-perforator group (1.4\%); however, this was not significant $(\mathrm{P}=0.53)$.

There were 4 cases of postoperative arterial thrombosis: 3 cases occurred in the 1-perforator group (2.0\%) and 1 case in the 2-perforator group (1.0\%) $(\mathrm{P}=0.7) .5$ cases of postoperative venous thrombosis were observed: 3 cases in the 1-perforator group (2.0\%) and 2 cases in the 2-perforator group (1.9\%) $(\mathrm{P}=0.75)$. These outcomes did not vary significantly among the cohorts.

Fat necrosis also did not differ significantly across cohorts, occurring clinically in 20 flaps overall (6.5\%). 15 flaps experienced this complication in the 1-perforator group (10.2\%), as compared to 2 flaps in the 2-perforator group (2.9\%), and 2 flaps in the 3-perforator group (4\%). While this did demonstrate a modestly greater rate of fat necrosis in flaps with 1 perforator, this difference did not reach statistical significance $(P=0.1)$.

We then conducted a subgroup analysis that divided our DIEP flaps into 1-perforator flaps versus multiple $(>1)$ perforator flaps to determine a potential association with flap loss or overall flap complications (Table 3). Of the 310 DIEP flaps, 147 (47.4\%) were 1-perforator flaps, while 163 (52.6\%) were multiple-perforator flaps. These cohorts were generally similar, but the 1-perforator flap cohort had a slightly higher BMI (29.3 vs. 28.4, P = 0.04).

Five flap losses occurred in the 1-perforator subgroup (3.4\%), while 3 flap losses occurred in the multiple-perforator subgroup (1.8\%) (Table 3). Although there was an almost 2-fold increase 
Table 4. Subgroup analysis by WHO obesity classification

\begin{tabular}{|c|c|c|c|c|c|}
\hline Characteristic & Non-obese $(n=204)$ & Class I $(n=64)$ & Class II $(n=32)$ & Class III $(n=7)$ & P-value \\
\hline Flap loss & $4(2.0)$ & $2(3.1)$ & $1(3.1)$ & $3(42.9)$ & $<0.001$ \\
\hline Fat necrosis & $18(8.8)$ & $7(10.9)$ & $3(3.1)$ & $0(0.0)$ & 0.88 \\
\hline
\end{tabular}

Table 5. Analysis of fat necrosis within WHO obesity cohort classifications

\begin{tabular}{|lccc|}
\hline Fat necrosis WHO & 1 Perforator & $>1$ Perforator & P-value \\
\hline 0 & $12(12.9)$ & $3(2.7)$ & 0.006 \\
I & $2(6.3)$ & $3(11.1)$ & 0.67 \\
II & $2(12.5)$ & $0(0.0)$ & 0.48 \\
III & 0 & 0 & N/A \\
\hline Values are presented as number (\%), unless otherwise noted. \\
WHO, World Health Organization.
\end{tabular}

in the flap loss rate in the 1-perforator subgroup, this difference did not reach statistical significance $(\mathrm{P}=0.39)$.

When intraoperative and postoperative arterial and venous thrombosis was categorized by these subgroups, no significant differences were noted. However, when we examined fat necrosis by subgroup, we noted 15 cases of fat necrosis in the 1-perforator subgroup (10.2\%) as compared to only 5 cases of fat necrosis in the multiple-perforator subgroup (3.1\%): an almost 3 -fold and significant difference $(\mathrm{P}=0.009)$.

Finally, in the post-hoc analysis, we classified DIEP flaps by BMI to examine a potential association between major flap complications (flap loss and fat necrosis) and increasing BMI (Table 4). This analysis demonstrated that patients with class III obesity (BMI > 40) who underwent DIEP flaps had a $42.9 \%$ risk of DIEP flap loss $(\mathrm{P}<0.001)$. However, in the examination of fat necrosis by the $\mathrm{WHO}$ obesity classification, no significant differences were noted $(\mathrm{P}=0.88)$. A further evaluation of fat necrosis by the number of perforators within the obesity cohorts (Table 5) demonstrated that non-obese patients who underwent 1-perforator flaps had a significantly higher rate of fat necrosis than nonobese patients undergoing > 1-perforator DIEP flaps $(12.9 \%$ vs. $2.7 \%, \mathrm{P}=0.006$ ). No other differences were noted in a comparison of outcomes within the obesity classification cohorts.

\section{DISCUSSION}

Microsurgical success is built upon reliable perfusion to the free flap. With the advances in free-flap breast reconstruction towards lowering abdominal donor site morbidity, questions can at times still arise regarding flap perfusion. To date, there is a paucity of literature that analyzes the impact of the perforator number on DIEP flap viability and overall flap complications. The few studies that have addressed this topic have looked at limited outcomes and have produced conflicting data. The goal of this study was to critically examine a consecutive series of DIEP flaps in order to clarify this association between the perforator number and the overall flap outcome. Further, we sought to determine the impact of obesity on DIEP flaps as obesity has become an increasingly important health-related issue and one that impacts a significant proportion of breast reconstruction patients. Our findings demonstrate that with appropriate attention to surgical detail, the DIEP number does not appear to impact flap survival. However, preserving a greater number of perforators may decrease the likelihood of postoperative fat necrosis. Furthermore, our results shed more light on the challenges that reconstructive surgeons face with respect to breast reconstruction in the morbidly obese patient.

Our current practice when harvesting a DIEP flap follows an intraoperative anatomic algorithm that allows us to assess perforator flaps in real-time. Assuming that an adequate superficial inferior epigastric system is not present, we proceed to raise the abdominal flap from the lateral to medial direction, identifying all perforators. If a single perforator is centrally located and has an artery of $1.5 \mathrm{~mm}$, palpable pulse, and an accompanying vein of $2 \mathrm{~mm}$, we will proceed with a 1-perforator DIEP flap dissection. If there appears to be no single, dominant perforator, we will then examine the medial and lateral row. If there exist 2-4 medium-size perforators (a dopplerable 1-mm artery with a less than 2-mm visible vein) in the same row that require little to no sacrifice of the rectus abdominis muscle, a multiple-perforator DIEP flap will be harvested. If no such perforator pattern exists, we instead include several medium-sized perforators that are closely situated and perform a very limited msf-TRAM $[6,14]$. This algorithm has proven to afford successful reconstructions with low rates of flap loss and low accompanying donor site morbidity $[14,15]$.

In the current study, we demonstrated that the number of perforators harvested with DIEP flaps does not significantly affect the flap loss rate. However, our subgroup analysis did suggest a nearly 2 -fold increase in the flap loss rate in the 1-perforator subgroup versus that in the multiple-perforator subgroup (3.4\% vs. $1.8 \%, \mathrm{P}=0.39$ ). While this trend did not reach significance, 
this potential association could be further clarified in the future by an examination of a larger cohort. Additionally, our primary analysis revealed that the number of perforators does not appear to be significantly associated with overall flap complications.

These findings are in stark contrast to those of a study performed by Gill et al. [12] in 2004 that retrospectively reviewed 758 DIEP flaps. Gill et al. [12] found a significant increase in flap complications when more than 1 perforator was harvested $(P=0.04)$. Their explanation for this finding is that when a few perforators are harvested, it is typically because they are large and supply a relatively rich vascular inflow to the flap. However, their study's aim was simply to compile a database of DIEP flap outcomes for future comparison purposes, whereas our study focuses primarily on the association between the perforator number and the DIEP flap viability and complications. However, it is important to realize that based upon our intraoperative algorithm, we would also perform a one-perforator DIEP secondary to the interpretation that one perforator is sufficiently large to supply the entire flap.

Our analysis also revealed that as the number of perforators increases, there is an inverse trend in the rate of fat necrosis. Although this did not reach statistical significance in our primary analysis, we demonstrated an almost 3-fold, significant increase in the rate of fat necrosis in the 1-perforator subgroup as compared to that in the multiple-perforator subgroup (10.2\% vs. $3.1 \%, \mathrm{P}=0.009$ ). This finding may suggest overall superior flap perfusion by multiple perforators, as a more robust blood supply is directed to the more peripheral areas of the flap.

The literature offers divergent findings regarding this relationship between the perforator number and the fat necrosis rate. Gill et al. also found an increase in the fat necrosis rate with an increasing number of perforators, although significance was not reached. A related study on this topic by Bauman et al. [8] analyzed the relationship between the perforator number and the fat necrosis rate in msf-TRAM flaps, DIEP flaps, and SIEA flaps. In their prospective analysis, Bauman et al. found that msfTRAM flaps, which typically incorporated 3-5 perforators in their study, had the lowest rate of fat necrosis at 5\%. DIEP flaps, which typically incorporated 1-2 perforators, had the highest rate of fat necrosis at $25 \%(\mathrm{P}=0.007)$. These results are more in line with our findings.

However, a recently published critique by Rozen et al. [16] did note inherent shortcomings in the Bauman study, namely that the number of perforators was counted intraoperatively instead of being based on angiographic imaging. This is similar to our intraoperative perfusion assessment. Further, Rozen et al. pointed out that the perforator diameter in Bauman's study was measured externally and did not take into account variable vessel wall thickness, which is a better indicator of blood flow through the vessel.

While the findings of Bauman et al. are in agreement with our own, their analysis is different, in that it focuses primarily on fat necrosis and its association with the perforator number, regardless of the flap type. In contrast, this current study examines the DIEP flaps alone, with flap viability and overall flap complications being our primary outcomes of interest in association with the perforator number.

Fat necrosis is a complication that adversely affects outcomes. It compromises the aesthetic result by causing firm nodules that feel unnatural, and it undermines the suppleness of the reconstructed breast. More concerning, fat necrosis can mimic recurrent breast cancer both on palpation and on imaging [8]. Our study suggests that harvesting additional perforators when feasible may help decrease the risk of this complication. When this is not feasible, foregoing the DIEP flap for the more robust blood supply of an msf-TRAM flap may be a better option. We accordingly take this into consideration while moving through the intraoperative algorithm.

Finally, in our post-hoc analysis, we found a significantly higher DIEP flap loss rate in the morbidly obese. There has been an increasing prevalence in morbid obesity (BMI $>40)$, which currently affects almost $5 \%$ of the US population [17]. With these growing figures, now more than ever, plastic and oncologic surgeons will be working together on breast reconstruction cases for the morbidly obese patient. Our results showed a concerning trend in flap loss with increasing obesity. Most notably, we report a $42.9 \%$ DIEP flap loss rate in morbidly obese patients. These findings are echoed in further analyses of free-flap reconstruction in the obese and morbidly obese [18-20]. Because of the high risk of flap loss in these patients, we recommend exercising extreme caution when utilizing DIEP flaps in morbidly obese patients. However, given the increased risk of donor site complications in this cohort, a challenging balance between perfusion and minimizing donor site morbidity must be struck. Interestingly, however, fat necrosis was not found to increase with an increase in obesity. Furthermore, the examination within WHO obesity classifications by perforator number isolated the significant increase in fat necrosis to be in the non-obese cohort who had 1-perforator DIEP flaps. No other differences were noted in this analysis, although it was certainly limited by the small numbers with morbid obesity.

This study is certainly not without significant limitations. First, this is a retrospective study that depends heavily on the accuracy of medical record keeping as opposed to intraoperative observation and is prone to observer bias. We obtained the number of perforators from operative notes dictated by different providers. 
Unfortunately, 85 DIEP flaps (21\%) did not have a recorded number of perforators in the operative report and as such were not included in the full analysis of the perforator number. However, we did compare this cohort to the cohort of flaps where the perforator number was known, and no significant differences were noted. As such, we felt that it was appropriate to continue with the analysis. Finally, a retrospective study does not use any control groups for the comparison. Thus, our analysis relies on the assumption that it is the difference in the number of perforators that accounts for the difference in flap outcomes as opposed to other confounding factors. For instance, we did not provide data on flap weight as it is not a recorded parameter in our database. This information may be important because the amount of tissue that needs to be irrigated by the perforator may affect the rate of fat necrosis in addition to the number of perforators. Lastly, given the intraoperative algorithm, our flaps are selected for success. However, this in some ways gives even stronger support to the analysis of perforator number, because the number was not randomly chosen but was based on an assessment of perfusion by experienced microsurgeons.

A further limitation concerns our post-hoc analysis. Because we did not plan for this analysis a priori, we were limited to analyzing the data that we had collected for our initial hypothesis. Thus, although we did note a significant increase in the flap loss rate with increasing BMI, it is important to note that our sample numbers were not uniform throughout our BMI categorizations. We had only 8 patients in our BMI $>40$ group. If an association between patient $\mathrm{BMI}$ and the flap loss rate is to be thoroughly investigated, a larger sample of morbidly obese patients undergoing DIEP flap breast reconstruction should be considered.

We conclude that the number of DIEP flap perforators does not significantly affect the flap loss rates when careful assessment of intraoperative perfusion is employed. However, the rate of fat necrosis may be higher in single-perforator DIEP flaps, suggesting that multiple perforators may lead to overall superior flap perfusion. Lastly, the use of DIEP flaps in a morbidly obese patient should be carefully considered, weighing the risks of flap-related complications to donor site complications postoperatively.

\section{REFERENCES}

1. Sisco M, Du H, Warner JP, et al. Have we expanded the equitable delivery of postmastectomy breast reconstruction in the new millennium? Evidence from the national cancer data base. J Am Coll Surg 2012;215:658-66.

2. Man LX, Selber JC, Serletti JM. Abdominal wall following free TRAM or DIEP flap reconstruction: a meta-analysis and critical review. Plast Reconstr Surg 2009;124:752-64.

3. Garvey PB, Buchel EW, Pockaj BA, et al. DIEP and pedicled TRAM flaps: a comparison of outcomes. Plast Reconstr Surg 2006;117:1711-9.

4. Selber JC, Nelson J, Fosnot J, et al. A prospective study comparing the functional impact of SIEA, DIEP, and muscle-sparing free TRAM flaps on the abdominal wall: part I. unilateral reconstruction. Plast Reconstr Surg 2010;126:1142-53.

5. Selber JC, Fosnot J, Nelson J, et al. A prospective study comparing the functional impact of SIEA, DIEP, and muscle-sparing free TRAM flaps on the abdominal wall: Part II. Bilateral reconstruction. Plast Reconstr Surg 2010;126:1438-53.

6. Nelson JA, Guo Y, Sonnad SS, et al. A Comparison between DIEP and muscle-sparing free TRAM flaps in breast reconstruction: a single surgeon's recent experience. Plast Reconstr Surg 2010;126:1428-35.

7. Kroll SS. Free TRAM or DIEP flap: which to choose. Oper Tech Plast Reconstr Surg 1999;6:83-5.

8. Baumann DP, Lin HY, Chevray PM. Perforator number predicts fat necrosis in a prospective analysis of breast reconstruction with free TRAM, DIEP, and SIEA flaps. Plast Reconstr Surg 2010;125:1335-41.

9. Chang DW, Wang B, Robb GL, et al. Effect of obesity on flap and donor-site complications in free transverse rectus abdominis myocutaneous flap breast reconstruction. Plast Reconstr Surg 2000;105:1640-8.

10. Schusterman MA, Kroll SS, Miller MJ, et al. The free transverse rectus abdominis musculocutaneous flap for breast reconstruction: one center's experience with 211 consecutive cases. Ann Plast Surg 1994;32:234-41.

11. Kroll SS. Fat necrosis in free transverse rectus abdominis myocutaneous and deep inferior epigastric perforator flaps. Plast Reconstr Surg 2000;106:576-83.

12. Gill PS, Hunt JP, Guerra AB, et al. A 10-year retrospective review of 758 DIEP flaps for breast reconstruction. Plast Reconstr Surg 2004;113:1153-60.

13. Scheer AS, Novak CB, Neligan PC, et al. Complications associated with breast reconstruction using a perforator flap compared with a free TRAM flap. Ann Plast Surg 2006;56: 355-8.

14. Fischer JP, Sieber B, Nelson JA, et al. Comprehensive outcome and cost analysis of free tissue transfer for breast reconstruction: an experience with 1303 flaps. Plast Reconstr Surg 2013;131:195-203.

15. Cleveland EC, Fischer JP, Nelson JA, et al. Optimizing the fascial closure: an analysis of 1261 abdominally based free flap reconstructions. Ann Plast Surg 2013;71:255-60. 
16. Rozen WM, Whitaker IS, Chubb D, et al. Perforator number predicts fat necrosis in a prospective analysis of breast reconstruction with free TRAM, DIEP, and SIEA flaps. Plast Reconstr Surg 2010;126:2286-8.

17. Flegal KM, Carroll MD, Ogden CL, et al. Prevalence and trends in obesity among US adults, 1999-2000. JAMA 2002; 288:1723-7.

18. Jandali S, Nelson JA, Sonnad SS, et al. Breast reconstruction with free tissue transfer from the abdomen in the morbidly obese. Plast Reconstr Surg 2011;127:2206-13.
19. Fischer JP, Nelson JA, Sieber B, et al. Free tissue transfer in the obese patient: an outcome and cost analysis in 1258 consecutive abdominally based reconstructions. Plast Reconstr Surg 2013; 131:681e-92e.

20. Fischer JP, Cleveland EC, Nelson JA, et al. Breast reconstruction in the morbidly obese patient: assessment of 30day complications using the 2005 to 2010 National Surgical Quality Improvement Program data sets. Plast Reconstr Surg 2013;132:750-61. 